\title{
The impact of social support on the risk of eating disorders in women exposed to intimate partner violence
}

This article was published in the following Dove Press journal:

International Journal of Women's Health

25 November 2015

Number of times this article has been viewed

\author{
Dana K Schirk' \\ Erik B Lehman ${ }^{2}$ \\ Amanda N Perry 3 \\ Rollyn M Ornstein ${ }^{4}$ \\ Jennifer S McCall- \\ Hosenfeld ${ }^{2,5}$ \\ 'The Pennsylvania State University \\ College of Medicine, ${ }^{2}$ Department \\ of Public Health Sciences, The \\ Pennsylvania State University College \\ of Medicine, Hershey, ${ }^{3}$ Department \\ of Agricultural Economics, Sociology, \\ and Education, Division of Rural \\ Sociology, College of Agriculture, \\ Pennsylvania State University, \\ University Park, ${ }^{4}$ Department of \\ Pediatrics, ${ }^{5}$ Department of Medicine, \\ The Pennsylvania State University \\ College of Medicine, Hershey, PA, USA
}

Correspondence: Jennifer S McCallHosenfeld

The Pennsylvania State University College of Medicine, 500 University Drive, Mail Code A2 10, Hershey, PA 17033, USA

$\mathrm{Tel}+\mathrm{I} 717$ 53| 8I6I

Fax +I 7175317726

Email jmccallhosenfeld@hmc.psu.edu
Background: Eating disorders (EDs) are often found among women exposed to intimate partner violence (IPV). The role of social support (SS) as a protective factor against ED among IPV-exposed women is not firmly established.

Objective: The objective of this study is to determine the distribution of risk of EDs among women exposed to IPV and to examine the impact of SS on risk of ED among IPV-exposed women.

Methods: Women (aged 18-64 years) exposed to IPV during their lifetimes (defined by the Humiliation-Afraid-Rape-Kick instrument) were recruited from primary care and domestic violence service agencies and surveyed on demographics, mood/anxiety disorders, psychosocial/ community factors, and strategies used in response to IPV. The Eating Disorder Screen for Primary Care assessed the risk of ED. A modified Medical Outcomes Study Social Support Survey assessed overall functional support (scale range: 0-32; categorized into quartiles). Ordinal logistic regression examined the risk of ED based on SS, controlling for prespecified demographics (age, race/ethnicity, marital status, near-poverty level), and health-related factors significant in bivariate analyses (risky alcohol use).

Results: Among 302 women with lifetime IPV, 41 (14\%) were at high risk, 127 (42\%) were at moderate risk, and 134 (44\%) were at low risk of an ED. In bivariate analyses, high risk of an ED was significantly more frequent among women with a low SS score $(<19,24 \%)$ versus a high SS score $(\geq 30,12 \%)(P=0.03)$. High risk of an ED was significantly associated with risky alcohol use $(18 \%)$ versus non-risky alcohol use $(13 \% ; P=0.008)$. In multivariable analysis, a 5 -unit increase in overall SS was significantly associated with decreased odds of ED risk $(P=0.007)$.

Conclusion: Among IPV-exposed women, low SS is associated with an increased risk of ED. SS may protect against ED by reducing anxiety and promoting positive actions, but further study is needed to confirm this.

Keywords: women, domestic violence, spouse abuse, social support, eating disorders

\section{Background}

Forty-four percent of women in the USA are exposed to intimate partner violence (IPV) during their lifetime. ${ }^{1}$ IPV, as defined by the Centers for Disease Control and Prevention (CDC), is physical, sexual, or psychological harm by a current or former spouse or partner. ${ }^{2}$ Physical harm is the intentional use of physical force with the potential of causing death, disability, or injury and may include punching, hitting, burning, slapping, and use of a weapon. Sexual harm includes rape, other forms of sexual coercion, and unwanted sexual contact. Psychological harm consists of insults, belittling, constant humiliation, intimidation, threats of harm, and threats to take away children. ${ }^{2}$ 
Women exposed to IPV have an increased risk of numerous adverse health conditions including sexually transmitted diseases and musculoskeletal and reproductive conditions, an increased risk of adverse health risk behaviors such as substance abuse and tobacco use, and an increased risk of adverse mental health diagnoses such as depression and anxiety. ${ }^{3}$ Eating disorders (EDs) are an important adverse mental health condition associated with IPV. In an Australian study, IPV was associated with a 1.87 relative risk of EDs. ${ }^{4}$ IPV is one of many independent risk factors in the multifactorial etiology of EDs. These risk factors have been found in several studies comparing patients with anorexia nervosa (AN) and bulimia nervosa $(\mathrm{BN})$ to controls and looking at many factors such as socioeconomic status, self-esteem, childhood experiences, abuse, mental health, and dieting habits. ${ }^{5}$

In North America, AN and BN affect $1.4 \%$ and $2.5 \%$ of women, respectively. ${ }^{6}$ Exposure to IPV may play an important role in the development of EDs. Situational stress from IPV may lead women to seek control in their eating habits causing an increased risk of EDs. ${ }^{7}$ A systematic review of eight studies that assessed for EDs using a validated instrument showed that EDs are associated with a high prevalence and increased odds of a lifetime history of IPV in both males and females $\geq 13$ years of age. ${ }^{8}$ Despite these findings, the authors of this systematic review found the data on EDs and IPV to be limited. Several important evidence gaps included whether the associations between EDs and IPV vary by type of IPV exposure and IPV timing. In addition, studies did not adequately control for factors that can influence the relationship between EDs and IPV, such as childhood abuse and comorbid psychiatric conditions. ${ }^{7}$ Thus, more research is needed to investigate the strength and nature of the association between IPV and EDs, as well as the additional factors that may impact this association.

Social support (SS) is one such factor that may mitigate the risk of EDs in women exposed to IPV. Increased SS correlates with better health outcomes - especially mental health outcomes - in other research among abused women. ${ }^{9}$ For example, among women separated from abusive partners, increased emotional SS positively impacted health, while social conflict negatively mediated the relationship between the severity of IPV and health. ${ }^{9}$ Likewise, among female drug users exposed to IPV, lower levels of personal emotional SS significantly correlated with drug use. ${ }^{10}$ Decreased incidence of suicide has been shown among African Americans exposed to partner abuse with higher levels of SS. ${ }^{11}$

Prior literature has examined the role of SS in patients diagnosed with EDs. A systematic review showed that SS is a protective factor that mitigates risk factors for EDs, such as body dissatisfaction. ${ }^{12}$ Patients with BN and AN have been found to have smaller social networks with less emotional and practical support in comparison to those without EDs. ${ }^{13}$ Based on the positive impact that increased SS has on other aspects of mental health, one might conclude that SS would reduce the likelihood of EDs among IPV-exposed women.

However, current research does not show a clear association between SS and EDs among IPV-exposed women. Perceived SS in college students who had experienced dating violence in the past month was found to buffer the relationship between eating disturbances and abuse, but symptomatic dieting and bulimic symptoms were not directly related to SS when depressive symptoms were controlled. ${ }^{14}$ Also, among sexually abused adolescents, as family support and parent-adolescent communication increased, purging behavior decreased. However, purging increased with more extra-familial adult support. ${ }^{15}$ Thus, further research is needed to examine the association between SS and EDs among IPV-exposed women.

In this study, we examined the frequency of risk of EDs in an adult population of women exposed to IPV, and then assessed the role of SS in the risk of EDs among these women. We hypothesized that women exposed to more recent IPV would have higher risk of EDs, and that SS would reduce the risk of ED among women exposed to IPV.

\section{Methods}

\section{Subjects}

All participants were women between the ages of 18 and 64 years. The two methods of recruitment are shown in Figure 1. All women, aged 18-64 years, who had an outpatient visit between October 1, 2011, and September 30, 2012, at a site served by the Penn State Ambulatory Research Network - a primary care network serving a diverse region of central Pennsylvania - were initially eligible. These 24,338 women were stratified by rurality of residence so that ruralresiding women could be oversampled to provide adequate geographic variation in the study sample. A random sample of 2,500 women was then selected from this population. These 2,500 women were sent a brief screening survey that asked questions about health conditions, demographics, health behaviors, habits such as substance use, and IPV exposure as measured by the Humiliation-Afraid-RapeKick (HARK) screener. HARK is a four-question screening instrument for IPV that has been validated in primary care settings, and accurately identifies women experiencing IPV; 


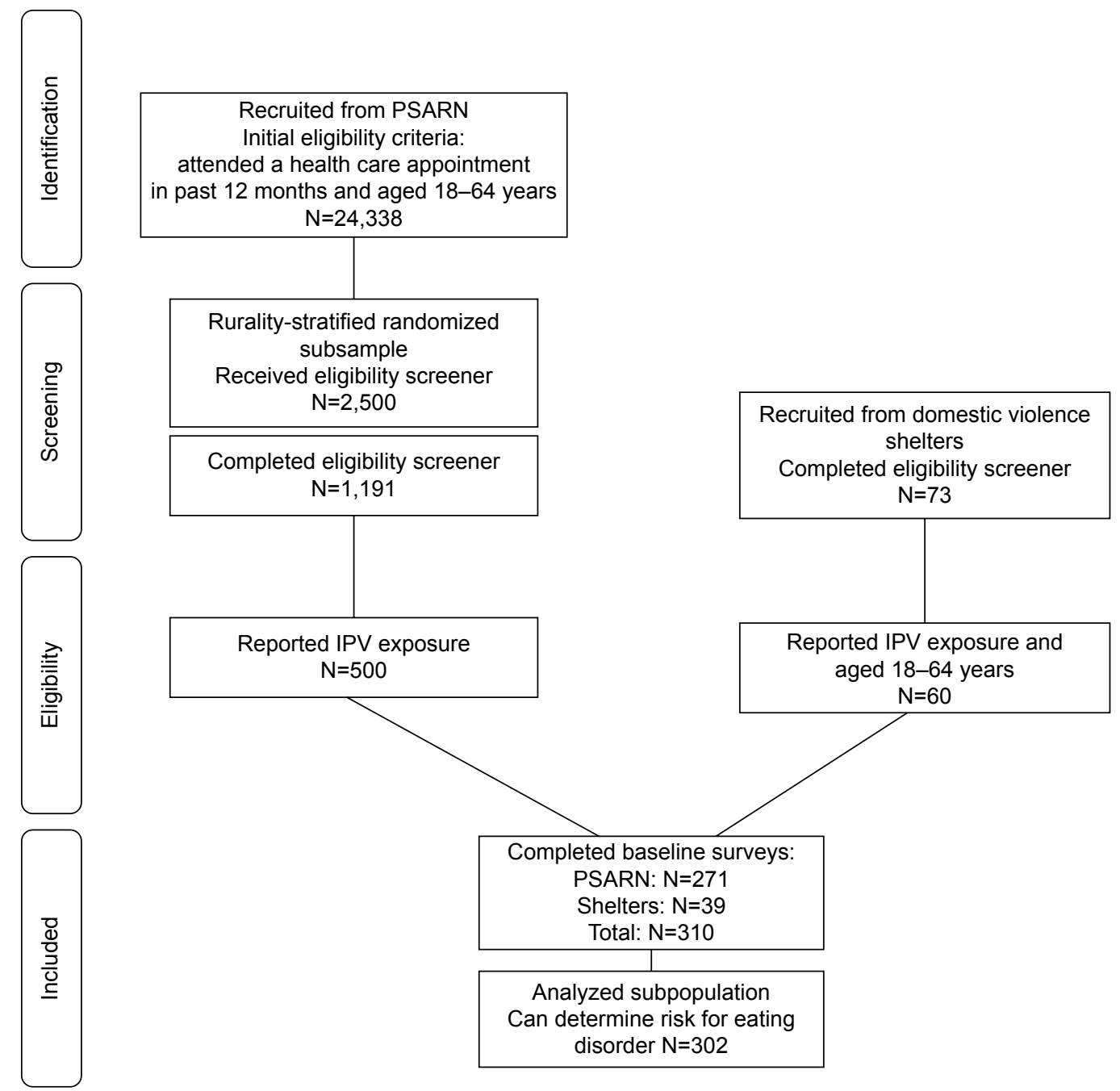

Figure I Recruitment of subjects.

Abbreviations: PSARN, Penn State Ambulatory Research Network; IPV, intimate partner violence.

it encompasses emotional, sexual, and physical abuse. ${ }^{16}$ The HARK questions are as follows:

- "Have you ever been humiliated or emotionally abused in other ways by your partner or your ex-partner?"

- "Have you ever been afraid of your partner or ex-partner?"

- "Have you ever been raped or forced to have any kind of sexual activity by your partner or ex-partner?"

- "Have you ever been kicked, hit, slapped, or otherwise physically hurt by your partner or ex-partner?"

The HARK screener was modified to ask about both recent (past 12 months) and lifetime IPV exposure. Following the screening questions, women were invited to leave their contact information if they wished to participate in further surveys. They were given the option to participate by returning the screening form via US postal service, entering the responses online, or contacting the investigators and reviewing the screening questions over the phone with study personnel. The screener was accompanied by a $\$ 2$ up-front incentive, and included consent information. Up to two reminder letters were sent to improve response rates to the screening survey. Surveys that were returned by mail were hand-entered by a study personnel with a second study personnel performing audit verification of the data entry.

A total of 1,191 women completed the eligibility screener (a 48\% response rate). Of these women, 500 reported IPV exposure based on HARK. Women who screened positive for lifetime IPV exposure and provided their contact information were invited to take part in a longer baseline survey. Of the women reporting IPV exposure who left their contact information, 271 ( $87 \%$ of the sample) consented and completed the longer survey, which asked more detailed information about relationships, physical and mental health, including the risk of EDs, sociodemographics, $\mathrm{SS}$, and responses to 
IPV exposure. We were unable to contact 74 women, seven refused to participate, and 34 never completed the survey.

Women were also recruited from 26 participating domestic violence agencies. Flyers were posted in these agencies serving rural and non-rural areas, inviting them to contact the investigators by phone or to take the screening survey online. Seventy-three women identified in this way completed the screener. Among these women, 60 reported IPV based on the HARK screener; 39 women (13\% of the sample) left their contact information, consented, and completed the baseline survey. We were unable to contact four women, one refused to participate, and seven never completed the survey.

In total, 310 out of 560 eligible women completed the baseline survey, giving a response rate of $55 \%$, which is a comparable response rate with other studies of IPV-exposed women. For example, a prior study reported a $56.6 \%$ response rate found among IPV-exposed women completing self-report telephone interviews. ${ }^{17}$ Variation in response rates between studies is likely attributable to differences in sampling methodology. Out of the 310 women completing the baseline survey, 302 fully completed responses on risk of EDs and formed the analytic sample for this study.

\section{Safety and confidentiality}

Participants received an informed consent document along with the eligibility screener and baseline survey. All forms of data collection clearly requested that participants indicate that they had reviewed the informed consent materials prior to participating in any part of the survey. If the surveys were completed by telephone, participants reviewed the consent document with trained study personnel and provided verbal consent prior to participating in the baseline survey. All consent documents clearly indicated that potential participants could choose not to participate in the study and could skip any questions they did not wish to answer. Approval was obtained from the Pennsylvania State University College of Medicine Institutional Review Board for the protocol and materials used for the study. To further protect the participants' safety and confidentiality, a Certificate of Confidentiality (CC-MH12204) was obtained from the National Institutes of Health for the project. To decrease the potential risks for participants, the screening surveys were identified as women's health surveys. After screening, potential participants who wished to proceed with the baseline survey were asked to review all consent-related documents and study documents in a safe, secure, and private location. Each survey also contained a list of resources related to the topics and health concerns mentioned in the survey, including referral information for services for general women's health, domestic violence, mental health, substance abuse, and EDs.

\section{Baseline survey}

Out of the 310 women completing the 30-minute to 45-minute baseline survey, 227 (73.7\%) did so online, 14 (4.55\%) via telephone, and $67(21.8 \%)$ by a mailed paper survey, based on their preference. The telephone surveys were conducted by trained interviewers. Data from the paper surveys were entered into the database by study staff, with audit verification by a second member of the study team. The baseline survey collected information about demographics, IPV status (type and lifetime exposure), mood and anxiety disorders, psychosocial and other health-related factors, and strategies that women used to address IPV. As before, the HARK screener was used to determine the type of IPV exposure the women had experienced and whether the IPV exposure had occurred in the past year. Table 1 lists the instruments used in the baseline survey along with the variable being examined, psychometric properties related to the variable if available, source, method of variable calculation, and an example question. The baseline survey was a self-report questionnaire. All participants who completed the baseline survey received a $\$ 25$ gift card.

Study data from both the screening survey and baseline survey were collected and managed using Research Electronic Data Capture $\left(\mathrm{REDCap}^{\mathrm{TM}}\right)$ tools hosted at Pennsylvania State University. ${ }^{18}$ REDCap $^{\mathrm{TM}}$ is a secure, Health Insurance Portability and Accountability Act-compliant, web-based application designed to support data capture for research studies, providing an intuitive interface for validated data entry, audit trails for tracking data manipulation and export procedures, automated export procedures for seamless data downloads to common statistical packages, and procedures for importing data from external sources. All baseline surveys were completed between May 29, 2013, and January 28, 2014.

\section{Outcome variable}

The Eating Disorder Screen for Primary Care (ESP) was used to measure the risk of EDs in women with IPV exposure. ${ }^{19}$ The ESP contains five yes/no questions that ask about satisfaction with eating patterns, eating in secret, how weight affects body image, family history of an ED, and past or present history of an ED. The ESP screener was developed as a brief screener for EDs from prior literature. ${ }^{20-22}$ The question "Does your weight affect the way you feel about yourself?" from the ESP screener prior was found 


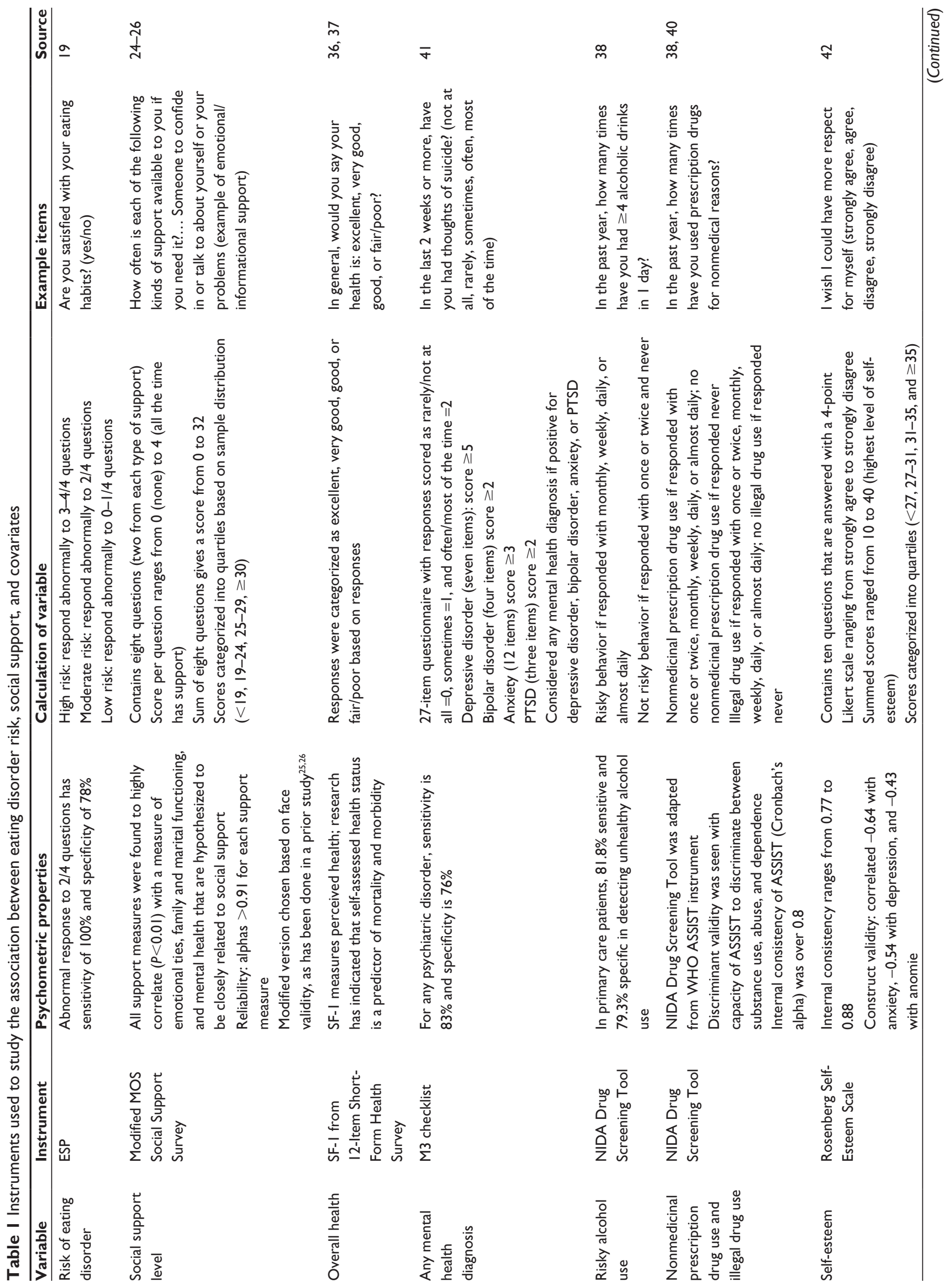




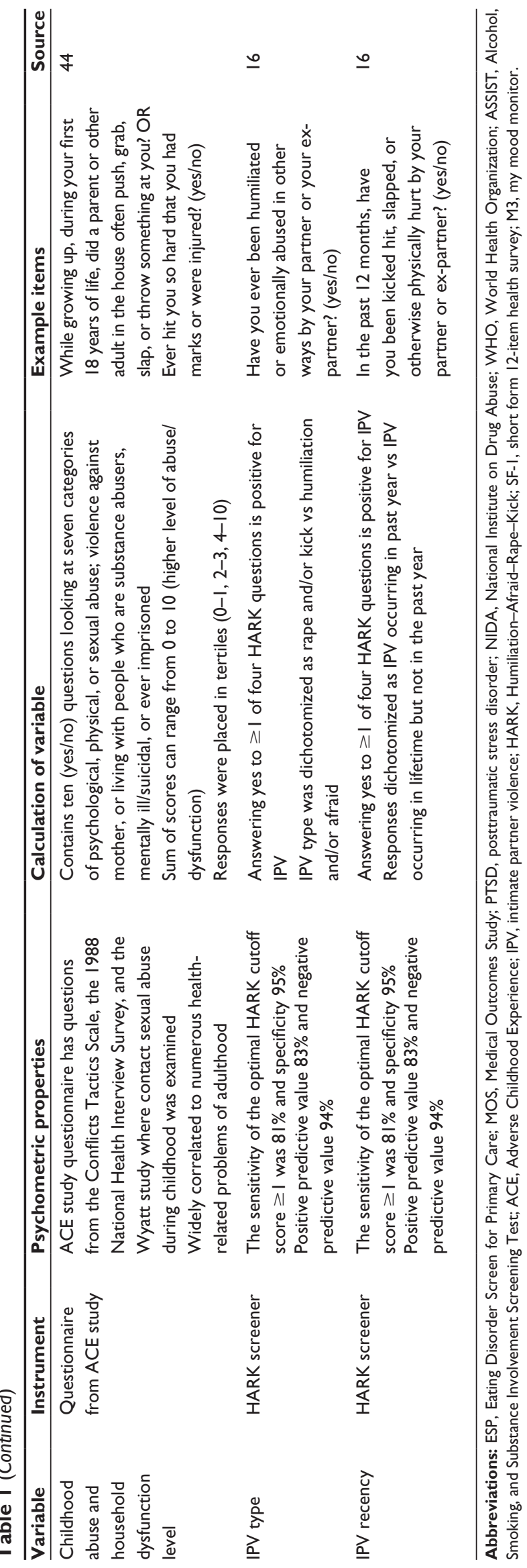

to have a sensitivity of $96.97 \%$ and specificity of $61.33 \%$ for disordered eating. ${ }^{20}$ The questions "Are you satisfied with your eating habits?" and "Do you ever eat in secret?" from the ESP screener were found to have $100 \%$ sensitivity and $90 \%$ specificity for detecting BN in a primary care setting. ${ }^{21}$ The ESP screener has been validated to broadly screen for the EDs: AN, BN, binge-eating disorder (BED), non-binging bulimia, and eating disorders not otherwise specified (EDNOS).$^{19}$ When excluding the question about family history of EDs in the ESP questions, the other four ESP questions (cutoff $\geq 2$, abnormal responses) were found to have a sensitivity of $100 \%$ and a specificity of $78 \%$ for the risk of an ED in college students and adults in a primary care setting. ${ }^{19}$ The four ESP questions were also found to better rule out an ED than the five-question Sick Control One Stone Fat Food (SCOFF) clinical prediction guide (sensitivity of $78 \%) .{ }^{23}$ In order to have a more extreme gradient of risk of ED to better analyze the association with SS, we created an ordinal variable in which zero to one abnormal response on the ESP was categorized as low risk of an ED, two abnormal responses were categorized as moderate risk of an ED, and three to four abnormal responses were categorized as high risk of an ED. These categorizations are based on natural splices in the data (Table 2).

\section{Exposure variable}

The baseline survey contained eight questions from the Medical Outcomes Study (MOS) Social Support Survey to assess the level of SS of the women exposed to IPV. The MOS Social Support Survey is a 19-item questionnaire that is organized by different types of SSs, including emotional/ informational support, tangible support, affectionate support, and positive social interactions. ${ }^{24}$ An example item and psychometric properties of the MOS survey are displayed in Table 1. As in prior work, ${ }^{25}$ eight questions (two questions from each type of support) of the MOS survey were included to reduce respondent burden. These eight items were chosen based on face validity, as has been done in a prior study, the Central Pennsylvania Women's Health Study

Table 2 Distribution of the number of abnormal responses to the ESP screener to determine the risk of an eating disorder

\begin{tabular}{lllll}
\hline $\begin{array}{l}\text { No of abnormal } \\
\text { responses }\end{array}$ & $\begin{array}{l}\text { Frequency } \\
(\mathbf{n})\end{array}$ & $\%$ & $\begin{array}{l}\text { Cumulative } \\
\text { frequency (n) }\end{array}$ & $\%$ \\
\hline 0 & 14 & 4.64 & 14 & 4.64 \\
1 & 120 & 39.74 & 134 & 44.37 \\
2 & 127 & 42.05 & 261 & 86.42 \\
3 & 38 & 12.58 & 299 & 99.01 \\
4 & 3 & 0.99 & 302 & 100.00 \\
\hline \multicolumn{5}{l}{ Abbreviation: ESP, Eating Disorder Screen for Primary Care. }
\end{tabular}


(CePAWHS). ${ }^{25,26}$ A 5-point Likert-type scale was used to determine how often a participant received the support. The summed SS score ranged from 0 to 32 with 32 being the highest level of SS. For bivariate analysis, SS was split into quartiles creating a categorical variable for increasing the level of support. ${ }^{26}$ For multivariable analyses, a continuous variable was used to describe the increasing levels of SS.

\section{Control variables}

Demographic variables were included in analyses if they were thought to be significantly associated with the risk of $\mathrm{ED}$, based on review of prior literature. ${ }^{27-30}$ Relevant demographics included age, race/ethnicity, marital status, and poverty status. Age was self-reported, and groups were split into quartiles: $18-34,35-45,46-53$, and 54-64 years old. Age was treated as a categorical rather than a linear variable because the risk of EDs is not equally distributed across age groups, ${ }^{27}$ and does not necessarily have a direct linear association with age. Race/ethnicity was defined by self-report. ${ }^{28,31}$ The population sample was heavily non-Hispanic white, and therefore, in analyses, participants were categorized as either non-Hispanic white or other race/ethnicity. Marital status was determined by self-report. ${ }^{31}$ Response items were categorized as divorced, separated, married, partnered, widowed, or single. For analyses, divorced/separated responses were combined, and widowed/single responses were combined, due to small numbers. To examine socioeconomic status, a near-poverty variable ( $125 \%$ of the poverty level) was created based on the participants' total combined income before taxes, number of household members, and 2013 poverty guidelines. ${ }^{32}$

Health-related factors and behaviors considered in bivariate analyses included overall health, any mental health diagnosis (depression, bipolar disorder, anxiety disorder, and posttraumatic stress disorders), substance use, and self-esteem, as past studies have shown a significant relationship between these factors and EDs. ${ }^{33-35}$ Overall health was measured by question 1 (SF-1) from a 12-Item Short-Form Health Survey. ${ }^{36}$ Overall health was described as excellent, very good, good, fair, or poor. For analyses, excellent/very good responses and fair/poor responses were combined due to low numbers in the most extreme categories. Psychometric properties and sample item of the SF-1 are included in Table 1. ${ }^{37}$ All questions for substance use were adapted from the National Institute on Drug Abuse (NIDA) Drug Screening Tool that was adapted by NIDA from several sources, ${ }^{38,39}$ including the NIDA-Modified Alcohol, Smoking, and Substance Involvement Screening Test (ASSIST). ${ }^{40}$
Risky alcohol use was established if in the past year, an individual reported having four or more drinks in 1 day on a monthly basis or more frequently. Illegal drug use or the use of prescription drugs for nonmedical reasons was determined if an individual identified use of these drugs at least once or twice in the past year. Mental health was assessed with the M3 checklist, ${ }^{41}$ a validated scale that measures depression, anxiety, bipolar disorder, and/or posttraumatic stress disorder. Psychometric properties of the M3 checklist, how the mental health variable was calculated, and sample items are provided in Table 1. The Rosenberg Self-Esteem scale ${ }^{42}$ is a ten-item scale with a 4-point Likert-type response format ranging from "strongly agree" to "strongly disagree". Scores were scaled with a summed self-esteem score ranging from 10 to 40 with 40 being the highest level of self-esteem. For bivariate analysis, the self-esteem variable was separated into quartiles to examine how gradations of self-esteem may affect the risk of an ED. More information about the Rosenberg Self-Esteem scale is provided in Table 1.

Type of IPV, recency of IPV, history of childhood abuse, and household dysfunction were social factors included in bivariate analysis due to past significant associations with EDs in the literature. ${ }^{7,843}$ Type of IPV was dichotomized as either experiencing rape and/or other physical violence by an intimate partner (both indicative of physical abuse) or experiencing humiliation by and/or fear of an intimate partner (both suggestive of emotional/mental abuse). ${ }^{16}$ Recency of IPV was dichotomized as IPV that had occurred within the past year versus IPV that had occurred in a participant's lifetime but not within the last year. ${ }^{16}$ Childhood abuse and household dysfunction were measured by a ten-item questionnaire from the Adverse Childhood Experience (ACE) study. ${ }^{44}$ The ACE study is one of the largest investigations conducted to assess the associations between childhood maltreatment and well-being later in life, and is a collaboration between the CDC and Kaiser Permanente's Health Appraisal Clinic in San Diego. Table 1 contains more information about the variable. Responses for the variable were split into tertiles based on the sample responses. The overall ACE scale is correlated with broad effects on adverse health outcomes including mental health. ${ }^{45,46}$

\section{Statistical methods}

All variables were summarized with frequencies and percentages or means, medians, and standard deviations. Bivariate analyses using ordinal logistic regression examined the association of the risk of EDs with SS and demographic variables as well as health, behavioral, and social factors hypothesized 
to have an effect on the risk of EDs. Odds ratios (ORs) were used to quantify the magnitude and direction of any significant associations. The proportional odds assumption for ordinal logistic regression was tested using the Score Test. Multivariable ordinal logistic regression assessed the odds of risk of EDs by SS after adjusting for all demographic and other variables found to be significantly associated with the risk of EDs in the bivariate analysis. Prior to the multivariable logistic regression, all independent variables were tested for multicollinearity using variance inflation factor statistics from linear regression; no multicollinearity was found. To assess the fit of the adjusted multivariable model, we used the Hosmer and Lemeshow Goodness-of-Fit test which was not significant demonstrating an adequate model fit. All analyses were carried out using SAS software version 9.4 (SAS Institute, Cary, NC, USA).

\section{Results}

Among 302 women with lifetime history of IPV, 41 (14\%) were at high risk, $127(42 \%)$ were at moderate risk, and 134 (44\%) were at low risk of an ED according to the ESP. In unadjusted bivariate analysis (Table 3), 12\% of women with a high level of overall functional support at a score $\geq 30$ had high risk of an ED, compared to $24 \%$ of women with a low level of SS at a score $<19$. The association between SS and risk of ED was statistically significant $(P=0.030)$.

Demographics including age, race/ethnicity, and nearpoverty status were not significantly associated with the risk of EDs (Table 3). Marital status was significantly associated with the risk of EDs $(P=0.033)$. The odds of high risk of an ED was higher in a married women than in women who were partnered but not married (OR $0.44,95 \%$ confidence interval [CI] 0.25-0.79). Risky alcohol use was significantly associated with high risk of an $\operatorname{ED}(P=0.008)$. Overall health, any mental health diagnosis, misuse of prescription drugs, illegal drug use, and self-esteem were not significantly associated with the risk of an ED. Additionally, level of childhood abuse and household dysfunction were not significantly associated with the risk of ED, nor was type, or recency of IPV (Table 3).

Our multivariable analysis, shown in Table 4, was prespecified to include SS, all demographic variables, and any health, behavioral, or social factors that were significant in bivariate analysis. Thus, while controlling for demographic variables and risky alcohol use, a 5-unit increase in overall SS showed a significant association with decreased odds of ED risk $(P=0.007)$. Marital status was significantly associated with the risk of an $\operatorname{ED}(P=0.026)$; a participant who was partnered, but not married, had a lower odds of ED risk than a married participant (OR 0.38; 95\% CI 0.20-0.72). Risky alcohol use was also significantly associated with a 2.39 increased odds of ED risk (95\% CI 1.3-4.4).

A cutoff $=2$ on the ESP is clinically considered to be high risk of an ED. Thus, in additional analyses, we also examined the association between the risk of ED using ESP $\geq 2$ and the previously defined SS and control variables. In unadjusted bivariate analysis, 59\% of women with a high level of overall functional support had high risk of an ED, compared to $61 \%$ of women with a low level of SS. Using this definition, the association between SS and ED risk trended in the same direction as our prior analysis but did not reach statistical significance $(P=0.110)$. In multivariable analysis, while controlling for demographic variables and risky alcohol use, a 5-unit increase in overall SS again showed a nonsignificant trend toward decreased odds of higher risk of an $\operatorname{ED}(P=0.095)$.

\section{Discussion}

EDs are among the top ten leading causes of disability among young women. ${ }^{47}$ In the USA, 20 million women suffer from a clinically significant ED at some time in their lives. ${ }^{48}$ In our study, we found $14 \%$ of women exposed to IPV to be at high risk of an ED. This frequency is substantially higher than the frequency of $\mathrm{AN}(1.4 \%)$ and $\mathrm{BN}(2.5 \%)$ in the general population, ${ }^{4,6}$ substantiating prior evidence of a strong link between lifetime IPV and ED risk. However, the ESP screener used to determine the risk of an ED does not just screen for $\mathrm{AN}$ and $\mathrm{BN}$ but also screen for BED, non-binging bulimia, and EDNOS, which could influence the higher frequencies found in our study. ${ }^{19}$ Past research has also revealed insufficient evidence to determine whether IPV recency or type more strongly correlates to the risk of an ED. ${ }^{7}$ Our study did not find a significant relationship between the type of IPV or IPV recency and risk of an ED. A small number of individuals with recent IPV or physical abuse, as well as homogeneity in the sample, ie, only women with lifetime IPV exposure, might account for this lack of statistical significance.

ED symptoms may develop as a negative coping mechanism in response to the situational stress of IPV. ${ }^{49}$ SS might mitigate the risk of EDs, but this relationship has not been clearly established. We found that among IPVexposed women, low SS was significantly associated with an increased risk of EDs, strongly suggesting that SS may be protective against EDs in IPV-exposed women.

Even though SS was found to be significantly associated with the risk of an ED, one factor possibly affecting the level of significance in this association is network orientation. 
Table 3 Bivariate analysis of overall functional social support, demographics, health, behavior, and social factors with the risk of eating disorder

\begin{tabular}{|c|c|c|c|c|c|c|}
\hline & $\begin{array}{l}\text { Total } \\
(\mathrm{N}=302)\end{array}$ & $\begin{array}{l}\text { Low risk } \\
\text { (score 0-I) }\end{array}$ & $\begin{array}{l}\text { Moderate } \\
\text { risk (score 2) }\end{array}$ & $\begin{array}{l}\text { High risk } \\
\text { (score 3-4) }\end{array}$ & $\begin{array}{l}\text { Odds ratio } \\
(95 \% \mathrm{Cl})\end{array}$ & $P$-value \\
\hline \multicolumn{7}{|c|}{ Overall functional social support } \\
\hline Quartile I $(<19)$ & 72 & $28(38.9)$ & $27(37.5)$ & $17(23.6)$ & Reference & $0.030^{*}$ \\
\hline Quartile 2 (19-24) & 85 & $35(4 \mid .2)$ & 39 (45.9) & II (I2.9) & $0.73(0.4 I-1.33)$ & \\
\hline Quartile 3 (25-29) & 70 & $40(57.1)$ & $26(37.1)$ & $4(5.7)$ & $0.38(0.20-0.73)^{*}$ & \\
\hline Quartile $4(\geq 30)$ & 68 & $28(4 \mid .2)$ & $32(47.1)$ & $8(11.8)$ & $0.72(0.38-1.34)$ & \\
\hline \multicolumn{7}{|l|}{ Demographics } \\
\hline \multicolumn{7}{|l|}{ Age (years) } \\
\hline $18-34$ & 80 & $35(43.8)$ & $36(45.0)$ & $9(11.3)$ & Reference & 0.949 \\
\hline $35-45$ & 75 & $34(45.3)$ & $28(37.3)$ & $13(17.3)$ & $1.08(0.59-1.95)$ & \\
\hline $46-53$ & 70 & $32(45.7)$ & $30(42.9)$ & $8(11.4)$ & $0.94(0.5 \mathrm{I}-1.74)$ & \\
\hline 54-64 & 76 & $32(42.1)$ & $33(43.4)$ & II (I4.5) & $1.12(0.62-2.03)$ & \\
\hline \multicolumn{7}{|l|}{ Race/ethnicity } \\
\hline Non-Hispanic white & 270 & $|2|(44.8)$ & II 4 (42.2) & $35(13.0)$ & $0.69(0.33-1.4 I)$ & 0.307 \\
\hline Other & 29 & II (37.9) & $12(4 \mid .4)$ & $6(20.7)$ & Reference & \\
\hline \multicolumn{7}{|l|}{ Marital status } \\
\hline Divorced/separated & 55 & $26(47.3)$ & $21(38.2)$ & $8(14.6)$ & $0.75(0.4|-| .35)$ & $0.033^{*}$ \\
\hline Married & 140 & $55(39.3)$ & $62(44.3)$ & $23(16.4)$ & Reference & \\
\hline Partnered & 66 & $38(57.6)$ & $25(37.9)$ & $3(4.6)$ & $0.44(0.25-0.79)^{*}$ & \\
\hline Widowed/single & 40 & $15(37.5)$ & $18(45.0)$ & $7(17.5)$ & $1.08(0.56-2.09)$ & \\
\hline \multicolumn{7}{|l|}{ Near poverty } \\
\hline Yes & 34 & $15(44.1)$ & $14(4 \mid .2)$ & $5(14.7)$ & $1.03(0.52-2.02)$ & 0.938 \\
\hline No & 349 & III (44.6) & $103(4 \mid .4)$ & $35(14.1)$ & Reference & \\
\hline \multicolumn{7}{|c|}{ Health and behavioral factors } \\
\hline \multicolumn{7}{|c|}{ Overall health } \\
\hline Excellent/very good & 127 & $54(42.5)$ & $57(44.9)$ & $16(12.6)$ & $0.70(0.37-1.33)$ & 0.248 \\
\hline Good & 126 & $60(47.6)$ & $52(4 \mid .3)$ & $14(11.1)$ & $0.58(0.31-1.10)$ & \\
\hline Fair/poor & 46 & $18(39.1)$ & $17(37.0)$ & II (23.9) & Reference & \\
\hline \multicolumn{7}{|c|}{ Any mental health diagnosis } \\
\hline Yes & 110 & $48(43.6)$ & $45(40.9)$ & $17(15.5)$ & $1.10(0.7|-| .7 \mid)$ & 0.674 \\
\hline No & 192 & $86(44.8)$ & $82(42.7)$ & $24(12.5)$ & Reference & \\
\hline \multicolumn{7}{|l|}{ Risky alcohol use } \\
\hline Yes & 56 & $15(26.8)$ & $31(55.4)$ & $10(17.9)$ & $2.10(1.21-3.65)^{*}$ & $0.008^{*}$ \\
\hline No & 244 & $118(48.4)$ & $95(38.9)$ & $31(12.7)$ & Reference & \\
\hline \multicolumn{7}{|c|}{ Nonmedicinal prescription drug use } \\
\hline Yes & 30 & $10(33.3)$ & $13(43.3)$ & $7(23.3)$ & $1.85(0.9 \mid-3.76)$ & 0.091 \\
\hline No & 270 & $124(45.9)$ & $112(4 \mid .5)$ & $34(12.6)$ & Reference & \\
\hline \multicolumn{7}{|l|}{ Illegal drug use } \\
\hline Yes & 17 & $8(47.1)$ & $6(35.3)$ & $3(17.7)$ & $1.00(0.40-2.53)$ & 0.997 \\
\hline No & 284 & $126(44.4)$ & $120(42.3)$ & $38(13.4)$ & Reference & \\
\hline \multicolumn{7}{|l|}{ Self-esteem } \\
\hline Quartile I (<27) & 69 & $27(39.1)$ & $26(37.7)$ & $16(23.2)$ & Reference & 0.271 \\
\hline Quartile 2 (27-30) & 66 & $29(43.9)$ & 27 (40.9) & $10(15.2)$ & $0.72(0.38-1.36)$ & \\
\hline Quartile $3(31-34)$ & 68 & $30(44.1)$ & $33(48.5)$ & $5(7.4)$ & $0.62(0.33-1.17)$ & \\
\hline Quartile $4(\geq 35)$ & 83 & $41(49.4)$ & $33(39.8)$ & $9(10.8)$ & $0.56(0.30-1.03)$ & \\
\hline \multicolumn{7}{|l|}{ Social factors } \\
\hline \multicolumn{7}{|c|}{ Childhood abuse and household dysfunction level } \\
\hline Tertile I (0-I) & 108 & $45(41.7)$ & $51(47.2)$ & $12(11.1)$ & Reference & 0.077 \\
\hline Tertile $2(2-3)$ & 86 & $45(52.3)$ & $33(38.4)$ & $8(9.3)$ & $0.69(0.40-1.19)$ & \\
\hline Tertile $3(4-10)$ & 95 & $37(39.0)$ & $39(4 I . I)$ & $19(20.0)$ & $1.31(0.78-2.21)$ & \\
\hline \multicolumn{7}{|l|}{ IPV type } \\
\hline Humiliation-afraid & 99 & $42(42.4)$ & $52(52.5)$ & $5(5.1)$ & Reference & 0.498 \\
\hline Rape-kick & 203 & $92(45.3)$ & $75(37.0)$ & $36(17.7)$ & I. $17(0.74-1.85)$ & \\
\hline \multicolumn{7}{|l|}{ IPV recency } \\
\hline Recent IPV & 78 & $36(46.2)$ & $26(33.3)$ & $16(20.5)$ & Reference & 0.637 \\
\hline Lifetime IPV & 224 & $98(43.8)$ & $101(45.1)$ & $25(11.2)$ & $0.89(0.55-1.45)$ & \\
\hline
\end{tabular}

Note: $* P$-values or odds ratios with $\mathrm{Cl}$ that show the variable is significantly associated with risk for an eating disorder. Abbreviations: $\mathrm{Cl}$, confidence interval; IPV, intimate partner violence. 
Table 4 Ordinal logistic regression model to predict the risk of eating disorder and overall functional social support, adjusting for demographics and risky alcohol use

\begin{tabular}{llll}
\hline & aOR & $95 \%$ Cl & $\operatorname{Pr}>\chi^{2}$ \\
\hline $\begin{array}{l}\text { Overall functional social } \\
\text { support (5-unit increase) }\end{array}$ & $0.79 *$ & $0.67-0.94^{*}$ & $0.007^{*}$ \\
Demographics & & & \\
$\quad$ Age (years) & & & 0.789 \\
$\quad$ 35-45 vs I8-34 & 0.77 & $0.40-1.48$ & \\
$\quad 46-53$ vs I8-34 & 0.72 & $0.36-1.45$ & \\
$\quad 54-64$ vs I8-34 & 0.76 & $0.38-1.51$ & \\
$\quad$ Non-Hispanic white: yes vs no & 0.79 & $0.37-1.72$ & 0.553 \\
$\quad$ Marital status & & & $0.026^{*}$ \\
$\quad$ Divorced/separated vs married & 0.68 & $0.35-1.31$ & \\
$\quad$ Partnered vs married & $0.38^{*}$ & $0.20-0.72^{*}$ & \\
$\quad$ Widowed/single vs married & 0.90 & $0.43-1.90$ & \\
$\quad$ Near-poverty: yes vs no & 0.79 & $0.40-1.56$ & 0.493 \\
Health and behavioral factors & & & \\
$\quad$ Risky alcohol use: yes vs no & $2.39 *$ & $1.31-4.37^{*}$ & $0.005^{*}$ \\
\hline
\end{tabular}

Note: $* P$-values or odds ratios with $\mathrm{Cl}$ that show the variable is significantly associated with risk for an eating disorder.

Abbreviations: aOR, adjusted odds ratio; $\mathrm{Cl}$, confidence interval; $\mathrm{Pr}$, probability.

Network orientation is an individual's willingness to utilize his or her SS. ${ }^{50,51}$ Our question about SS asked, "How often is each of the following kinds of support available to you if you need it?" We did not assess whether women utilized the support that was available. Negative network orientation is indicative of an individual's belief about the inadvisability, impossibility, uselessness, or potential danger of drawing on network resources. ${ }^{50,51}$ Women exposed to IPV may exhibit negative network orientation, ie, choose not to use their SS due to feelings of shame, fear, embarrassment, and concern for safety of themselves or their children..$^{52}$ Therefore, negative network orientation may have impacted the utility of SS among participants as it may not accurately reflect whether they were actually using the support available to them. If IPV-exposed women had SS that they were unable to access, this could have minimized the impact of such SS on the risk of EDs.

In our study, among all the control variables examined, risky alcohol use was found to be significantly associated with an increased ED risk. This finding supports past research including a study which found that alcoholic women had a $35 \%$ rate of a previous or current ED. ${ }^{34}$ The rate of $\mathrm{BN}$ and alcohol use disorders co-occurring in clinical and community samples has been well documented,${ }^{53}$ and one study in particular found that $49 \%$ of BN patients used alcohol several times or more per week. ${ }^{54}$ This frequency is substantially $>3.5 \%$ of women who reported daily alcohol use in a nationwide survey ${ }^{55}$ or $8.45 \%$ of the general US population having any alcohol use disorder. ${ }^{56}$ There are many theories as to why there is a co-prevalence between EDs and alcohol use.
The relationship may be due to the presence of an addictive personality, shared vulnerability for addiction to endogenous and exogenous substances, family history of substance use, vulnerability to adolescent stressors, need for self-medication for depression, and/or anxiety. ${ }^{57}$ However, these hypotheses have not clarified the relationship between EDs and substance use and have not been supported sufficiently or consistently with empirical evidence. ${ }^{57}$ Age, race/ethnicity, marital status, and near-poverty were chosen as control variables because in past studies, they were found to have a relationship with the risk of EDs. ${ }^{27-29,31}$ However, in our study, there was no significant relationship between these demographics and risk of an ED. EDs tend to be first present in adolescents, as well as young adults in their late teens to $20 \mathrm{~s}^{27}$ Limited numbers of young adults in our sample may have affected our ability to discern a significant association between the age and risk of EDs. Our population was mostly non-Hispanic white, a race/ethnicity category that has been found to have higher rates of $\mathrm{BN}$ and $\mathrm{AN} .{ }^{28}$ The limited sample of Hispanic, nonwhite individuals limited our ability to examine ethnicity/ race as a risk factor for $\mathrm{ED}$. Although the association between AN and high socioeconomic status remains to be adequately proven, BN may be related to a lower socioeconomic status. ${ }^{31}$ However, our sample may not have had sufficient economic diversity to determine an association between economic status and ED risk.

In our study, we found that ED risk was associated with being married versus partnered but not married. This is contrary to prior literature showing that marriage is associated with significant decreases in disordered eating from late adolescence to midlife. ${ }^{29}$ Our finding may be related to the fact that our married population were also exposed to IPV; thus, married, IPV-exposed women may be particularly vulnerable to EDs, perhaps due to negative coping.

Our data had several limitations and strengths to consider. First, the population tested in the study lacked ethnic diversity, and the results might not be applicable to a sample that is more racially or ethnically diverse. The data are also crosssectional, and therefore, causality cannot be established. Additionally, the ESP screener was previously validated only in a small primary care and college settings that had a high prevalence of disordered eating, and it has not been examined in a wide variety of cultural or age groups, which may limit its broad applicability. The use of only self-report questionnaires without structured interviews may represent another limitation. Self-report questionnaires are easy to administer and inexpensive, but information derived from self-report can be potentially subject to response bias such 
as imprecise recall and the influence of social desirability. This response bias can mask or distort the true underlying relationships between variables. ${ }^{58}$ Some of the other common method biases from self-report questionnaires include mood state, which is the propensity of respondents to view themselves in negative terms (negative affectivity) or in positive terms (positive affectivity). Another potential problem with self-report questionnaires is consistency motif, in which respondents show a propensity to maintain consistency in their responses to questions and common scale formats such as Likert scales, thereby creating artifactual covariation. ${ }^{59}$

Strengths of the study include the fact that the ED and SS screeners could be easily used in a primary care setting, and are likely to accurately identify these risk factors. The survey examined a wide variety of demographic, health-related, and social factors. This allowed us to not only appreciate the association of SS and the risk of EDs but also ascertain the other factors significantly associated with EDs in IPVexposed women. Another strength of the study was the use of an ordinal variable (in bivariate analyses) and a continuous variable (in multivariable analyses) for determining ED risk, which allowed for detection of finer gradations in the association between SS and risk for ED. Additionally, the ESP detects the risk of a broad variety of EDs, which may not have been examined in other studies.

More research is needed to determine if improvement of SS could either prevent or ameliorate EDs among IPVexposed women, as suggested by our data. Future studies could replace the ESP screener with questions that screen for disordered eating versus EDs. Disordered eating can include specific behaviors such as eating too quickly, too little, and too irregularly to more global conditions like food restriction, $\mathrm{BN}$, and $\mathrm{AN} .{ }^{60}$ Disordered eating is broader and may be more strongly associated with SS. Additionally, future studies could examine specific eating behavior such as purging to determine whether there is a significant association with SS.

\section{Conclusion}

Among IPV-exposed women, a low level of SS was found to be significantly associated with high risk of an ED. IPV is a nonspecific risk factor for numerous adverse mental health consequences including EDs. ${ }^{61}$ Nevertheless, it is important to highlight IPV exposure as a risk factor for EDs so that clinicians who treat women with EDs may also be aware of IPV history and incorporate trauma-informed care into their treatment. Further study examining the extent to which IPVexposed women use the SSs available to them is needed to better understand these findings.
Future directions for this research could include examination of interventions to improve SS, such as support groups or improved access to health care, to determine if these interventions improve the health of women exposed to IPV and decrease their risk of EDs. Additionally, treatment for women with EDs who have a known exposure to IPV may benefit from strategies to improve SS systems. Internetdelivered interventions aimed at reducing risk factors for EDs may serve as a low-cost, easily accessible service for women with EDs. ${ }^{62,63}$ These interventions could be catered to women with EDs and exposure to IPV. However, to be effective, these interventions are likely to require assurances that IPV-exposed women access and utilize the support available to them.

\section{Acknowledgments}

Funding for this study was provided by a Clinical Scientist Development Award from the Doris Duke Charitable Foundation (grant number 2012064; principal investigator: Dr McCall-Hosenfeld). Study data were collected and managed using REDCap ${ }^{\mathrm{TM}}$ electronic data capture tools hosted at Penn State (Pennsylvania State University CTSI, NIH/NCATS grant number UL1 TR000127). Preliminary data from this work were previously presented, in part, as a poster at Women's Health 2014: The 22nd Annual Congress, Washington, DC, on 4/5/2014, and are available as an abstract in the conference proceedings. The authors thank their technical consultants at the Pennsylvania Coalition Against Domestic Violence. The authors assume full responsibility for the collection, analysis, and interpretation of this data; no funder or consultant participated in these efforts.

\section{Disclosure}

The authors report no conflicts of interest in this work.

\section{References}

1. Thompson RS, Bonomi AE, Anderson M, et al. Intimate partner violence: prevalence, types, and chronicity in adult women. Am J Prev Med. 2006; 30(6):447-457.

2. Saltzman LE, Fanslow JL, McMahon PM, Shelley GA. Intimate Partner Violence Surveillance: Uniform Definitions and Recommended Data Elements, Version 1.0. Atlanta, GA: Centers for Disease Control and Prevention, National Center for Injury Prevention and Control; 2002.

3. Bonomi E, Anderson M, Reid R, Rivara F, Carrell D, Robert T. Medical psychosocial diagnoses in women with history of intimate partner violence. Arch Intern Med. 2009;169:1692-1697.

4. Vos T, Astbury J, Piers LS, et al. Measuring the impact of intimate partner violence on the health of women in Victoria, Australia. Bull World Health Organ. 2006;84(9):739-744

5. Streigel-Moore R. Risk factors for eating disorders. Ann NY Acad Sci. 2006;817(1):98-109. 
6. Striegel-Moore R, Dohm F, Taylor B, Daniels S, Crawford P, Schreiber G. Eating disorders in white and black women. Am J Psychiat. 2003;160(7):1326-1331.

7. Campbell J, Campbell D. Disordered eating among African Americans and African Caribbean women: the influence of intimate partner violence, depression, and PTSD. Issues Ment Health Nurs. 2012;33:513-521.

8. Bundock L, Howard LM, Trevillion K, Malcolm E, Feder G, Oram S. Prevalence and risk of experiences of intimate partner violence among people with eating disorders: a systematic review. JPsychiat Res. 2013; 47(9):1134-1142.

9. Guruge S, Ford-Gilboe M, Samuels-Dennis J, Varcoe C, Wilk P, Wuest J. Rethinking social support and conflict: lessons from a study of women who have separated from abusive partners. Nurs Res Pract. 2012;2012:1-10.

10. Panchanadeswaran S, El-Bassel N, Gilbert L, Wu E, Chang M. An examination of the perceived social support levels of women in methadone maintenance treatment programs who experience various forms of intimate partner violence. Women Health Iss. 2008;18(1):35-43.

11. Kaslow NJ, Thompson MP, Meadows LA, et al. Factors that mediate and moderate the link between partner abuse and suicidal behavior in African American women. J Consult Clin Psych. 1998;66:533-540.

12. Stice E. Risk and maintenance factors for eating pathology: a metaanalytic review. Psychol Bull. 2002;128(5):825-848.

13. Tiller JM, Sloane G, Schmidt U, Troop N, Power M, Treasure JL. Social support in patients with anorexia nervosa and bulimia nervosa. Int $J$ Eat Disord. 1997;21(1):31-38.

14. Skomorovsky A, Matheson K, Anisman H. The buffering role of social support perceptions in relation to eating disturbances among women in abusive dating relationships. Sex Roles. 2006;54(9-10):627-638.

15. Perkins DF, Luster T. The relationship between sexual abuse and purging: findings from community-wide surveys of female adolescents. Child Abuse Negl. 1999;23:371-382.

16. Sohal H, Eldridge S, Feder G. The sensitivity and specificity of four questions (HARK) to identify intimate partner violence: a diagnostic accuracy study in general practice. BMC Fam Pract. 2007;8:49.

17. Thompson R, Bonomi A, Anderson M, et al. Intimate partner violence: prevalance, types, and chronicity in adult women. Am J Prev Med. 2006;30(6):447-457.

18. Harris PA, Taylor R, Thielke R, Payne J, Gonzalez N, Conde J. Research electronic data capture (REDCap) - a metadata-driven methodology and workflow process for providing translational research informatics support. J Biomed Inform. 2009;42(2):377-381.

19. Cotton MA, Ball C, Robinson P. Four simple questions can help screen for eating disorders. J Gen Intern Med. 2003;18:53-56.

20. Anstine D, Grinenko MD. Rapid screening for disordered eating in college-aged females in the primary care setting. $J$ Adolesc Health. 2000;26:338-342.

21. Freund KM, Graham SM, Lesky LG, Moskowitz MA. Detection of bulimia in a primary care setting. J Gen Intern Med. 1993;8:236-242.

22. McNulty PAF. Prevalence and contributing factors of eating disorder behaviors in active duty navy men. Mil Med. 1997;162:753-759.

23. Morgan JF, Reid F, Lacey H. The SCOFF questionnaire: assessment of a new screening tool for eating disorders. BMJ. 1999;319:1467-1468.

24. Sherbourne CD, Stewart AL. The MOS Social support survey. Soc Sci Med. 1991;32(6):705-714.

25. Weisman CS, Hillemeir MM, Chase GA, et al. Preconceptional health: risks of adverse pregnancy outcomes by reproductive life stage in the Central Pennsylvania Women's Health Study (CePAWHS). Women Health Iss. 2006;16(4):216-224.

26. Hillemeier M, Weisman C, Chase G, Dyer AM. Mental health status among rural women of reproductive age: findings from the Central Pennsylvania Women's Health Study. Am J Public Health. 2008;98(7): 1271-1279.

27. Hudson JI, Hiripi E, Pope HG, Kessler RC. The prevalence and correlates of eating disorders in the National Comorbidity Survey Replication. Biol Psychiat. 2007;61:348-358.
28. Striegel-Moore RH, Dohm FA, Kraemer HC, et al. Eating disorders in white and black women. Am J Psychiatry. 2003;160(7):1326-1331.

29. Keel PK, Baxter MG, Heatherton TF, Joiner TE Jr. A 20-year longitudinal study of body weight, dieting, and eating disorder symptoms. J Abnorm Psychol. 2007;116(2):422-432.

30. Gard MCE, Freeman CP. The dismantling of a myth: a review of eating disorders and socioeconomic status. Int J Eat Disord. 1996;20(1): $1-12$.

31. Salganicoff A, Beckman JZ, Wyn R, Ojeda VD. Women's Health in the United States: Health Coverage and Access to Care. Report by The Henry J Kaiser Foundation; May 2002.

32. 2013 Poverty Guidelines. Washington DC: U.S. Department of Health \& Human Services. Available from: http://aspe.hhs.gov/ poverty/13poverty.cfm. Accessed July 1, 2013.

33. Padierna A, Quintana JM, Arostegui I, Gonzalez N, Horcajo MJ. Changes in health related quality of life among patients treated for eating disorders. Qual Life Res. 2002;11:545-552.

34. Gadalla T, Piran N. Co-occurrence of eating disorders and alcohol use disorders in women: a meta-analysis. Arch Womens Health. 2007;10: 133-140.

35. Joiner GW, Kashubeck S. Acculturation, body image, self-esteem, and eating-disorder symptomatology in adolescent Mexican American women. Psychol Women Quart. 1996;20(3):419-435.

36. Ware J, Kosinski M, Keller SD. A 12-item short-form health survey: construction of scales and preliminary tests of reliability and validity. Med Care. 1996;34(3):220-233.

37. Gill T, Broderick D, Avery J, Grande E, Taylor A. Self-reported overall health status: implications for intervention strategies. Australas Med $J$. 2009;1(8):44-57.

38. Smith PC, Schmidt SM, Allensworth-Davies D, Saitz RA. Single-question screening test for drug use in primary care. Arch Intern Med. 2010; 170(13):1155-1160.

39. U.S. Department of Health and Human Services. National Institute on Alcohol Abuse and Alcoholism's Helping Patients Who Drink Too Much: A Clinician's Guide Updated 2005 Edition. Available from: http://pubs.niaaa.nih.gov/publications/practitioner/ CliniciansGuide2005/guide.pdf. Accessed June 1, 2013.

40. World Organization (WHO) alcohol, smoking, and substance involvement screening test (ASSIST), Version 3.0. Available from: http://www. drugabuse.gov/nmassist/. Accessed October 8, 2015.

41. Gaynes BN, DeVeaugh-Geiss J, Weird S, et al. Feasibility and diagnostic validity of the M-3 checklist: a brief, self-rated screen for depressive, bipolar, anxiety, and post-traumatic stress disorders in primary care. Ann Fam Med. 2010;8(2):160-169.

42. Rosenberg M. Rosenberg Self-Esteem Scale. Society and the Adolescent Self-Image. Princeton, NJ: Princeton University Press; 1965.

43. Wonderlich SA, Brewerton TD, Jocic Z, Dansky Bonnie S, Abbott DW. Relationship of childhood sexual abuse and eating disorders. $\mathrm{J} \mathrm{Am} \mathrm{Acad}$ Child Adolesc Psychiatry. 1997;36(8):1107-1115.

44. Felitti VJ, Anda RF, Nordenberg D, et al. Relationship of childhood abuse and household dysfunction to many of the leading causes of death in adults: the adverse childhood experiences (ACE) study. Am J Prev Med. 1998;14(4):245-258.

45. Edwards VJ, Holden GW, Anda RF, Felitti VJ. Experiencing multiple forms of childhood maltreatment and adult mental health: results from the Adverse Childhood Experiences (ACE) Study. Am J Psychiat. 2003; 160(8):1453-1460.

46. Anda R, Felitti V. Origins and Essence of the Study. ACE Reporter. 2003;1(1):1-4.

47. Striegel-Moore RH, Bulik CM. Risk factors for eating disorders. Am Psychol. 2007;62(3):181-198.

48. Wade TD, Keski-Rahkonen A, Hudson JI. Textbook of Psychiatric Epidemiology. 3rd ed. London: John Wiley \& Sons; 2011.

49. Friedman MM, King KB. The relationship of emotional and tangible support to psychological wellbeing among older women with heart failure. Res Nurs Health. 1994;17:433-440. 
50. Vaux A, Burda P, Stewart D. Orientation toward utilization of support resources. J Community Psychol. 1986;14:159-170.

51. Clapp J, Beck J. Understanding the relationship between PTSD and social support: the role of negative network orientation. Behav Res Ther. 2008;47(3):237-244.

52. Sylaska KM, Edwards KM. Disclosure of intimate partner violence to informal social support network members: a review of the literature. Trauma Violence Abus. 2014;15(3):1-19.

53. Dansky B, Brewerton T, Kilpatrick D. Comorbidity of bulimia nervosa and alcohol use disorders: results from the National Women's study. Int J Eat Disord. 2000;27:180-190.

54. Mitchell JE, Hatsukami D, Eckert ED, Pyle RL. Characteristics of 275 patients with bulimia. Am J Psychiat. 1985;142:482-485.

55. Johnston LD, O'Malley PM, Bachman JG. Use of Licit and Illicit Drugs by America's High School Students, 1975-1984. Rockville, MD: U.S. Dept. of Health and Human Services; 1985. [DHHS Publication No. ADM 85-1394, 159].

56. Grant B, Stinson F, Dawson D, et al. Prevalence and co-occurrence of substance use disorders and independent mood and anxiety disorders: results from the national epidemiologic survey on alcohol and related conditions. JAMA Psychiat. 2004;61(8):807-816.

57. Wolfe WL, Maisto SA. The Relationship between eating disorders and substance use: moving beyond co-prevalence research. Clin Psychol Rev. 2000;20(3):617-631.
58. Celis-Morales C, Perez-Bravo F, Ibanez L, Salas C, Bailey M, Gill J. Objective vs self-reported physical activity and sedentary time: effects of measurement method on relationships with risk biomarkers. PLoS One. 2012;7(5):1-10.

59. Podsakoff P, MacKenzie S, Jeong-Yeon P. Common method biases in behavioral research: a critical review of the literature and recommended remedies. J Appl Psychol. 2003;88(5):879-903.

60. Matthews M, Zullig KJ, Ward RM, Horn T, Huebner ES. An analysis of specific life satisfaction domains and disordered eating among college students. Soc Indic Res. 2012;107:55-69.

61. Pico-Alfonso M, Linares I, Navarro N, Ros C, Martinez E. The impact of physical, psychological, and sexual intimate male partner violence on women's mental health: depressive symptoms, posttraumatic stress disorder, state anxiety, and suicide. J Womens Health. 2006;15(5): 599-611.

62. McCormack A. Individuals with eating disorders and the use of online support groups as a form of social support. Comput Inform Nurs. 2010; 28(1):12-19.

63. Celio AA, Winzelberg AJ, Wilfley DE, et al. Reducing risk factors for eating disorders: comparison of an internet and a classroom-delivered psychoeducational program. J Consult Clin Psychol. 2000;68(4): 650-657.
International Journal of Women's Health

\section{Publish your work in this journal}

The International Journal of Women's Health is an international, peerreviewed open-access journal publishing original research, reports, editorials, reviews and commentaries on all aspects of women's healthcare including gynecology, obstetrics, and breast cancer. The manuscript management system is completely online and includes

\section{Dovepress}

a very quick and fair peer-review system, which is all easy to use. Visit http://www.dovepress.com/testimonials.php to read real quotes from published authors. 